\title{
Fas-mediated pathway and apoptosis in normal cervix, cervical intraepithelial neoplasia and cervical squamous cancer
}

\author{
JIAN-HONG ZHOU ${ }^{1}$, HUAI-ZENG CHEN ${ }^{2}$, FENG YE ${ }^{2}$, WEI-GUO LU ${ }^{1}$ and XING XIE ${ }^{1}$ \\ ${ }^{1}$ Department of Gynecologic Oncology, ${ }^{2}$ Central Laboratory, Women's Hospital, School of Medicine, \\ Zhejiang University, 2 Xueshi Road, Hangzhou 310006, China
}

Received January 2, 2006; Accepted February 23, 2006

\begin{abstract}
The aim of this study was to examine the expression patterns of apoptosis-related antigens, such as Fas, FasL, and cFLIP, in cervical squamous cells, and investigate the role of Fas-mediated apoptosis in the pathogenesis of cervical neoplasia. Using specific antibodies for Fas, FasL, and cFLIP, we examined protein expression in 19 specimens of normal cervix, 15 mild dysplasia (CIN I), 22 moderate dysplasia (CIN II), 23 severe dysplasia (CIN III), and 34 invasive squamous cell carcinoma (SCC) by immunohistochemistry. We detected the apoptotic indices by TUNEL in the same specimens. Fas expression levels were quite similar in CIN I, CIN II and the normal cervix. Though Fas expression tended to increase in grade 2 cancer compared to grade 1 cancer and CIN III, and a slight decline was present in grade 3 compared with grade 2 cancer, these differences did not reach statistical significance. Almost all CINs did not express FasL, while FasL expression increased with the grade of the tumor. Statistically significant differences could be observed between grade 1 and grade $2(\mathrm{p}<0.01)$ and between grade 2 plus grade 3 and grade $1(\mathrm{p}<0.001)$. All cases of normal cervix and CIN, except two cases of CIN III, did not express cFLIP. cFLIP expression tended to increase with the grade of the tumor. Apoptosis was determined in all samples by TUNEL. There was a decreasing tendency of apoptotic cells from normal cervix to cancers. A negative correlation between cFLIP and apoptosis ( $r=-0.499$ and $\mathrm{p}=0.000)$ was observed. Deregulated Fas/FasL system and constitutive expression of cFLIP in SCC may be an important mechanism by which SCCs escape apoptosis during the malignant transformation and progression of these tumors.
\end{abstract}

\section{Introduction}

A prerequisite for neoplastic cells to expand their population and eventually form solid tumors is the development of

Correspondence to: Dr Xing Xie, Department of Gynecologic Oncology, Women's Hospital, School of Medicine, Zhejiang University, 2 Xueshi Road, Hangzhou 310006, China

E-mail: xiex@mail.hz.zj.cn

Key words: Fas, FasL, cFLIP, apoptosis, cervical neoplasm strategies for escaping surveillance by the host immune system. The Fas-Fas ligand (FasL) pathway figures prominently in the execution of apoptosis induced by the immune system. Fas (CD95/APO-1) and its ligand (FasL/CD178) are cell surface molecules of the tumor necrosis factor receptor family. Fas is expressed in normal and neoplastic cells (1), and FasL expression is largely restricted to the immune system. On contact with FasL-positive cytotoxic lymphocytes, Fas-bearing tumor cells rapidly undergo apoptosis. However, tumors may mount a counterattack by expressing FasL, which represents a mode of tumor escape from immune surveillance (3). Effective utilization of the Fas system by tumor cells in fighting or defending cytotoxic lymphocytes would also require resistance to Fas-mediated cytotoxicity. Thus, a decreased susceptibility to apoptosis has indeed appeared to play a role in tumor survival (4) and viral immune escape (5).

The development of anti-apoptotic strategies by tumor cells is a prerequisite for overcoming autocrine suicidal elimination resulting from autologous Fas-FasL ligation. Cellular FLICElike inhibitory protein (cFLIP) has been identified as an endogenous inhibitor of apoptosis by virtue of its capability to bind to the death effector domain of Fas-associated death domain protein, a signal transducer of Fas-mediated apoptosis (6). Since tissue homeostasis is a delicate balance between proliferation and apoptosis, abnormal regulation of cFLIP expression may contribute to tumorigenesis (7).

In this study, we compared the indices of apoptosis and expression patterns of apoptosis-related antigens, Fas, FasL and c-FLIP in the normal cervix, cervical intraepithelial neoplasia (CIN) and cervical squamous cancer. In particular, we investigated the role of Fas-mediated apoptosis in the pathogenesis of cervical neoplasia.

\section{Materials and methods}

Cervical specimens. The study subjects were enrolled between November 2002 and April 2004 at the Center of Clinical Sample Resource Library, Women's Hospital, School of Medicine, Zhejiang University. None of the patients received chemotherapy or radiotherapy before surgery. A total of 94 patients with cervical lesions were enrolled, including 15 with mild dysplasia (CIN I) aged 26-64 years, 22 with moderate dysplasia (CIN II) aged 30-52 years, and 23 with severe dysplasia (CIN III) aged 26-71 years. Of 34 SCCs, 21 were stage IB and 13 were stage IIA; histopathologically, 10 were 
Table I. Expression of Fas, FasL, cFLIP and TUNEL score in the normal cervix, cervical intraepithelial neoplasia, and cervical squamous cell carcinoma.

\begin{tabular}{lccccc}
\hline Group & Total no. & Fas $^{\mathrm{a}}$ & FasL $^{\mathrm{a}}$ & cFLIP $^{\mathrm{a}}$ & TUNEL $^{\mathrm{b}}$ \\
\hline NC & 19 & $2.00(4.00)^{\mathrm{c}}$ & $2.00(4.00)^{\mathrm{d}}$ & $0.00(0.00)^{\mathrm{e}}$ & $3.50 \pm 0.77^{\mathrm{f}}$ \\
CIN & 60 & $2.00(3.00)$ & $0.00(3.50)$ & $0.00(1.50)$ & $3.08 \pm 1.82$ \\
CIN I & 15 & $0.00(3.00)$ & $0.00(0.00)$ & $0.00(0.00)$ & $3.90 \pm 1.08$ \\
CIN II & 22 & $2.00(3.00)$ & $0.00(2.50)$ & $0.00(0.00)$ & $3.45 \pm 0.79$ \\
CIN III & 23 & $3.00(4.00)$ & $0.00(4.00)$ & $0.00(4.00)$ & $2.35 \pm 1.15$ \\
SCC & 34 & $4.00(6.00)$ & $2.00(4.00)$ & $4.00(3.75)$ & $1.03 \pm 1.17$ \\
Grade 1 & 10 & $3.00(6.50)$ & $0.00(1.00)$ & $4.00(6.50)$ & $0.82 \pm 0.58$ \\
Grade 2 & 14 & $6.00(4.50)$ & $2.00(2.25)^{\mathrm{g}}$ & $3.50(3.25)$ & $1.43 \pm 0.85$ \\
Grade 3 & 10 & $3.00(2.50)$ & $4.00(2.50)$ & $6.00(5.75)$ & $0.72 \pm 0.64$
\end{tabular}

$\mathrm{NC}$, normal cervix; CIN, cervical intraepithelial neoplasia; SCC, cervical squamous cell carcinoma. ${ }^{\mathrm{a}} \mathrm{IS} \mathrm{M}\left(\mathrm{Q}_{\mathrm{R}}\right)$. ${ }^{\mathrm{b}} \mathrm{Per} 100$ epithelial cells $(\overline{\mathrm{x}} \pm \mathrm{s}) .{ }^{\mathrm{c}} \mathrm{NC}$ vs. $\mathrm{CIN}, \mathrm{p}<0.01$; CIN vs. SCC, $\mathrm{p}<0.001 .{ }^{\mathrm{d}} \mathrm{CIN}$ vs. SCC, $\mathrm{p}<0.01 .{ }^{\mathrm{e}} \mathrm{NC}$ vs. CIN, $\mathrm{p}<0.05$; NS vs. SCC, $\mathrm{p}<0.001 ; \mathrm{CIN}$ vs. SCC, $\mathrm{p}<0.001$. ${ }^{\mathrm{NC}}$ vs. CIN III, grade 1 , grade 2 , grade $3, \mathrm{p}<0.001$; CIN I vs. CIN III, grade 1 , grade 2 , grade 3 , p $<0.001$; CIN II vs. CINI II, grade 1 , grade 2, grade $3, \mathrm{p}<0.001$; CIN III vs. grade 1 , grade 2 , grade $3, \mathrm{p}<0.005$. ' Grade 1 vs. grade $2, \mathrm{p}<0.01$; grade 1 vs. grade 3 , $\mathrm{p}<0.001$; grade 1 vs. grade $2+$ grade $3, p<0.001$.

grade 1,14 were grade 2 and 10 were grade 3 . The samples were collected in $10 \%$ buffered formalin and embedded in paraffin within $24 \mathrm{~h}$ for long-term preservation.

To obtain cytologically normal controls, 19 normal uterine cervical epithelia were obtained from patients undergoing a hysterectomy for various nonmalignant diseases. All of the original hematoxylin-and-eosin-stained slides were blindly reviewed by two experienced pathologists.

Immunohistochemistry. The staining procedure strictly followed the guidelines of the Powervision test kit. Briefly, adjacent sections of $4-\mu \mathrm{m}$ thickness were cut and placed on poly-L-lysine-coated glass slides, and air-dried overnight at $60^{\circ} \mathrm{C}$. The sections were dewaxed in xylene and rehydrated in graded ethanol of $100 \%, 95 \%, 85 \%$, and $75 \%$ for $5 \mathrm{~min}$ each, and then rinsed in water. Slides were incubated for $30 \mathrm{~min}$ in $3 \%$ hydrogen peroxide in methanol to quench endogenous peroxidase activity and then pressure cooked for $2 \mathrm{~min}$ for antigen retrieval in $0.01 \mathrm{M}$ citric acid buffer. Immunostaining methods varied, depending on the antibody used. Ready-to-use Fas mouse monoclonal antiserum (ZM0295; Beijing Zhongshan Golden Bridge Biotechnology Co., Ltd.) was incubated overnight at $4^{\circ} \mathrm{C}$. Antibody binding was detected using a commercially available EnVision Plus-HRP kit (Dako, Dakopatts, Glostrup, Denmark) for 2 h. FasL rabbit polyclonal antibody (N-20, sc-843; Santa Cruz, CA) was used at a dilution of 1:50 and incubated for $2 \mathrm{~h}$. The cFLIPS/L rabbit polyclonal antibody (H-202, sc-8347; Santa Cruz) was used at a dilution of 1:80 and incubated overnight at $4^{\circ} \mathrm{C}$. The latter two antibody bindings were detected with EnVision ${ }^{\mathrm{TM}}$ rabbit peroxidase (Gene Tech Biotechnology Company Ltd.) for $1 \mathrm{~h}$. All three antibodies were developed by DAB substrate (3,3-diaminobenzidine tetrahydrochloride), and the sections were eventually counterstained with Harris' hematoxylin. Appropriate validation (negative controls) was performed by replacing the primary antibody with PBS alone.
Evaluation of IHC staining results. The scoring method of Sinicrope et al was applied to evaluate the IHC staining intensity and the proportion of positively stained epithelial cells (8). Membrane and nuclear staining were independently considered. The staining intensity was subclassified as 1 , weak; 2 , moderate; and 3, strong. The number of positive cells was expressed as the percentage of the total number of epithelial cells and assigned to one of five categories: $0,<5 \%$; $1,5-25 \% ; 2,26-50 \% ; 3,51-75 \%$; and $4,>75 \%$. The percentage of positive-stained tumor cells and the staining intensity were multiplied to produce an immunoreactive score (IS) for each specimen. Each lesion was examined and scored separately by two pathologists at the same time under the same conditions.

TUNEL and evaluation of apoptosis. To detect apoptotic cells in 4- $\mu$ m-thick sections of $10 \%$ formaldehyde-fixed and paraffin-embedded tissue, the in situ DNA nick-end labeling method (cat. no. 1684817; Roche, Germany) was used. All sections were mounted on poly-L-lysine-coated slides and deparaffinized. To facilitate the penetration of enzymes and biotinylated deoxyuridine into the nuclei, the slides were digested with proteinase $\mathrm{K}$ by incubating them in DNAasefree proteinase $\mathrm{K}(20 \mu \mathrm{g} / \mathrm{ml})$ for $20 \mathrm{~min}$ at room temperature. The slides were then immersed in terminal deoxynucleotidyl transferase enzyme (Tdt) labeling buffer for $5 \mathrm{~min}$. DNA fragments generated during apoptosis were end-labeled with modified nucleotides using a highly purified Tdt in a labeling buffer with cobalt chloride. Subsequently, in situ end-labeling of the fragmented DNA was performed by incubating the slides with $\mathrm{Tdt} /$ biotinylated deoxyuridine triphosphate diluted in Tdt buffer in a humidified chamber at $37^{\circ} \mathrm{C}$ for $1 \mathrm{~h}$. The slides were then immersed in a Tdt stop buffer for $5 \mathrm{~min}$ at room temperature, rinsed in PBS for $5 \mathrm{~min}$, and incubated with avidin-biotin peroxidase complex for $15 \mathrm{~min}$. The slides were then developed by using diaminobenzidine hydrogen peroxide. In negative controls, slides were incubated in Tdt buffer without the Tdt or biotinylated dUTP. The preparations 


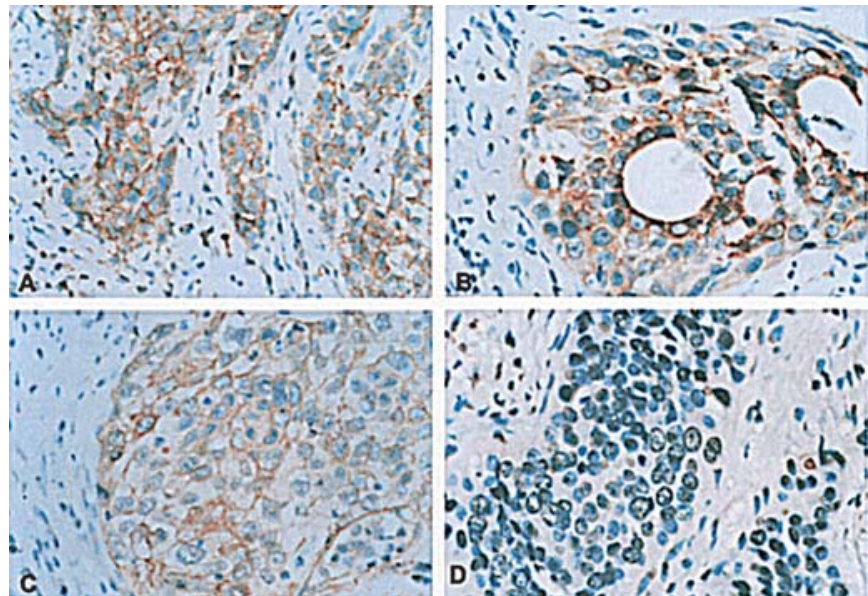

Figure 1. (A) Strong Fas positivity is seen in cervical squamous cell carcinoma. (B) Strong FasL-positive staining is present in cervical squamous cell carcinoma. (C) Strong membrane staining of cFLIP is seen in cervical squamous cell carcinoma. (D) Positive nuclear staining of TUNEL is seen in cervical squamous cell carcinoma. Original magnification, $\mathrm{x} 400$.

were counterstained with $0.3 \%$ methyl green solution. The apoptotic labeling index was calculated as percentages after counting the number of stained nuclei per 1000 cells under a light microscope at a x400 magnification. Apoptotic cells were identified by positive nuclear staining in combination with the typical histomorphological characteristics of apoptosis.

Statistical analysis. Statistical analyses were performed using SAS 8.0. To compare the means of immunoreactive scores, the Kruskal-Wallis test or one-way ANOVA was used. Associations between numeric variables were tested using the Spearman rank correlation coefficient. The Bonferroni test was used as the post-hoc test. A p-value $<0.05$ was regarded as statistically significant.

\section{Results}

Expression of Fas and FasL in cervical cancer. Results from the immunohistochemical analysis are summarized in Table I, and examples of typical staining are shown in Fig. 1. Fas showed clear membrane staining, but FasL appeared to be present in intracellular granules. In normal squamous epithelium, Fas was mainly expressed near the surface as expected and FasL, in contrast, was expressed predominantly in the basal layers. In many SCCs, very strong Fas expression appeared in the center of tumor areas often associated with keratinization, whereas FasL was expressed peripherally. Fas and FasL expression were seen in almost all tumor samples, although it varied considerably in both intensity and distribution. IS of Fas were quite similar in CIN I, CIN II and the normal cervix. Though Fas expression tended to increase in grade 2 cancer compared to grade 1 cancer and CIN III, and a slight decline was present in grade 3 compared with grade 2 cancer, these differences did not reach statistical significance. Almost all cases of CIN did not express FasL, while FasL expression increased with the grade of the tumor. Statistically significant differences were observed between grade 1 and grade 2, and between grade 2 plus grade 3 and grade 1 .
Expression of cFLIP and apoptosis. All cases of normal cervix and CIN except two cases of CIN III did not express cFLIP. cFLIP expression increased during the process of tumorigenesis from normal cervix to CINs and carcinoma. cFLIP expression tended to increase with the grade of the tumor, but no significant differences were observed between grade 1 and grade 2, or between grade 2 and grade 3 . Apoptosis was determined in all samples by TUNEL. There was a decreasing tendency of apoptotic cells from normal cervix to cancers. Statistically significant differences were observed between normal cervix and CIN III, and between CIN III and grade 1 , grade 2 and grade 3 .

Correlation between expression of Fas, FasL, cFLIP and apoptosis. When correlation among all four markers was evaluated in SCC, a positive correlation between Fas and $\mathrm{cFLIP}(\mathrm{r}=0.423$ and $\mathrm{p}=0.000)$ and a negative correlation between cFLIP and apoptosis $(\mathrm{r}=-0.499$ and $\mathrm{p}=0.000)$ were observed.

\section{Discussion}

Once the neoplastic process has settled, cell proliferation exceeds cell death. However, in the early stage of carcinogenesis, putative cell regulatory mechanisms come into play with increased apoptosis temporarily balancing cell proliferation (9). In our study, no apoptotic change was observed in CIN I and CIN II compared with normal cervix, while apoptosis decreased in CIN III and SCCs.

The regulation of apoptosis involves complex interactions among several proteins, including bcl-2, bax, and Fas (9-11). Since Fas expression was shown to be altered in a variety of many other neoplasms $(11,12)$, we assumed that Fas-mediated apoptosis might also be involved in cervical carcinogenesis. Fas is a membrane protein that belongs to the nerve growth factor receptor family, and its interaction with its ligand is one of the essential events in the induction of apoptosis $(11,13)$. When engaged with its ligand, Fas receptor undergoes trimerization, which in turn recruits caspase-8, activates the caspase cascade $(10,14)$ and initiates cell apoptosis. In the present study, we observed Fas positivity in normal cervix, CINs and SCCs, but there was a tendency towards increasing Fas expression in SCCs. However, Fas expression levels were similar in the CIN III and carcinoma groups, probably because CIN III lies close to carcinoma in the neoplasiacarcinoma spectrum. These two observations led us to suggest a role for Fas in early cervical carcinogenesis. Deregulated Fas and FasL expression were shown to also be involved in early esophageal carcinogenesis (5). Fas overexpression has been found in neoplasms, including esophageal, oral, hepatocellular, thyroid, and lung carcinomas (12,16-18). In our study, Fas expression was stronger and more diffuse in grade 2 carcinomas than grade 3 carcinomas. Muraki et al observed a similar relationship between Fas expression and tumor grade, with Fas being positive in well- and moderately welldifferentiated oral carcinomas, and negative in poorly differentiated types (18). Shibakita et al found similar results in esophageal carcinomas and suggested that downregulated Fas expression enabled tumor cells to survive host immune attack (16). Thus, Fas and FasL-mediated apoptosis might serve as a proofreading mechanism in human neoplasia. 
Upregulation of Fas receptors is possibly a defense mechanism of the host tissue against the neoplastic process. As the tumor grade increases, tumor cells might develop strategies to abrogate Fas receptor expression, and resist the apoptotic mechanism. As a result, apoptotic mechanisms that are functional in low-grade tumors fail in high-grade neoplasms, and this explains why we found lower levels of Fas in high-grade carcinomas than in low-grade carcinomas.

FasL is expressed on activated cytotoxic T cells and NK cells that have the ability to kill tumor cells by activating the Fas receptor on the latter. Thus, if tumor cells downregulate the expression of Fas, they might become less sensitive to FasLmediated killing by host immune cells. Conversely, FasL expression on the surface of tumor cells might lead to the death of Fas-sensitive infiltrating lymphocytes. In our study, FasL expression significantly increased with the grade of carcinoma. Thus, poorly differentiated carcinoma acquires an immune privilege. Membranous FasL plays a vital role in immune escape. Although the precise mechanism of Fas/FasLinduced apoptosis during the development of cervical cancer is unknown, studies of other tumor types, including melanomas (19) and hepatocellular carcinomas (3), have indicated that these tumors use Fas/FasL to induce apoptosis of infiltrating lymphocytes akin to immune privilege, which has been aptly termed 'tumor counterattack.'

cFLIP is one of the most extensively studied inhibitors of Fas-mediated apoptosis, which interacts with Fas-associated death domain (FADD) to form a complex that prevents cleavage of procaspase- 8 in the apoptotic cascade (20). Since tissue homeostasis is a delicate balance between proliferation and apoptosis, abnormal regulation of cFLIP expression may contribute to tumorigenesis (7). Several previous studies documented that increased expression of cFLIPs contributed to tumor resistance to Fas-mediated apoptosis, resulting in the escape of tumor cells from T-cell immunity, and was possibly related to tumor development, growth, and metastasis (21-23). In addition, it was previously shown that high expression levels of FLIP were sufficient to render sensory neuronal cells expressing moderate to low levels of Fas resistant to Fas-mediated death (24). Although the precise physiological role of cFLIP in malignant squamous cervical cancer is unknown to date, it is conceivable from this study that the significantly increased cFLIP expression in all cases of SCC probably plays a key role in counterattacking Fasmediated apoptosis by host immune cells and promoting subsequent tumor growth. Furthermore, we also demonstrated that cFLIP expression tended to increase with the grade of the tumor. In addition, a negative correlation between cFLIP and apoptosis $(r=-0.499$ and $\mathrm{p}=0.000)$ was observed. Therefore, our speculation that constitutive expression of cFLIP by SCC could be an important mechanism by which SCCs escape apoptosis during malignant transformation and progression of these tumors has been confirmed. Collectively, these results might allow speculation that the overexpression of cFLIP is involved in the process of malignant transformation.

The current study demonstrated that the malignant transformation of cervical epithelia is associated with abnormal expression of the Fas/FasL system. SCCs may counterattack immune cells by downregulation of Fas and killing lymphocytes through the expression of FasL. Furthermore, the overexpression of cFLIP in SCCs and a negative correlation between cFLIP and apoptosis appear to be an important mechanism by which SCCs escape apoptosis during the malignant transformation and progression of these tumors.

\section{Acknowledgements}

We thank Haiyan Shi and Xiaoduan Chen for their excellent pathological support.

\section{References}

1. Itoh N, Yonehara S, Ishii A, Yonehara M, Mizushima S, Sameshima M, Hase A, Seto Y and Nagata S: The polypeptide encoded by cDNA for human cell surface antigen Fas can mediate apoptosis. Cell 66: 233-243, 1991.

2. Suda T, Takahashi T, Golstein P and Nagata S: Molecular cloning and expression of the Fas ligand, a novel member of the tumor necrosis factor family. Cell 75: 1169-1178, 1993.

3. Strand S, Hofmann WJ, Hug H, Muller M, Otto G, Strand D, Mariani SM, Stremmel W, Krammer PH and Galle PR: Lymphocytic apoptosis induced by CD95 (APO-1/Fas) ligandexpressing tumor cells: a mechanism of immune evasion? Nat Med 2: 1361-1366, Biol Chem 270: 3255-3260, 1995.

4. Wright SC, Zhong J, Zheng H and Larrick JW: Inhibition of apoptosis as a mechanism of tumor promotion. FASEB J 8: 654-660, 1994.

5. Tewari $M$ and Dixit VM: Fas- and tumor necrosis factorinduced apoptosis is inhibited by the poxvirus crmA gene product. J Biol Chem 270: 3255-3260, 1995.

6. Nicholson DW and Thornberry NA: Caspases: killer proteases. Trends Biochem Sci 22: 299-306, 1997.

7. Budd RC: Death receptors couple to both cell proliferation and apoptosis. J Clin Invest 109: 437-441, 2002.

8. Sinicrope FA, Ruan SB, Cleary KB, Stephens C, Lee JJ and Levin B: bcl-2 and p53 oncoprotein expression during colorectal tumorigenesis. Cancer Res 55: 237-241, 1995.

9. Lu Q-L, Abel P, Foster CS and Lalani E-N: bcl-2: role in epithelial differentiation and oncogenesis. Hum Pathol 27: 102-110, 1996

10. Stadelman C and Lassmann H: Detection of apoptosis in tissue sections. Cell Tissue Res 301: 19-31, 2000.

11. Watanabe H, Kanzaki H, Narukawa S, Inoue T, Katsuragawa H, Kaneko Y and Mori T: Bcl-2 Fas expression in eutopic and ectopic human endometrium during menstrual cycle in relation to endometrial cell apoptosis. Am J Obstet Gynecol 176: 360-368, 1997.

12. Lee SH, Shin MS, Lee HS, et al: Expression of Fas and Fasrelated proteins in human hepatocellular carcinoma. Hum Pathol 32: 250-256, 2001.

13. Kase S, Osaki M, Adachi H, Kalibara N and Ito H: Expression of Fas and the Fas ligand in esophageal tissue mucosa and carcinomas. Int J Oncol 20: 291-297, 2002.

14. Kornmann M, Ishiwata T, Kleeff J, Beger HG and Korc M: Fas and Fas-ligand expression in human pancreatic cancer. Ann Surg 231: 368-379, 2000.

15. Gratas C, Tohma Y, Barnas C, Taniere P, Hainaut P and Ohgaki M: Up-regulation of Fas (APO/CD95) ligand and downregulation of Fas expression in human esophageal cancer. Cancer Res 58: 2057-2062, 1998.

16. Shibakita M, Tachibana M, Dhar DK, Kotoh T, Kinugasa S, Kubota H, Masunaga R and Nagasue N: Prognostic significance of Fas and Fas ligand expression in human esophageal cancer. Clin Cancer Res 5: 2464-2469, 1999.

17. Basolo F, Fiore L, Baldanzi A, Giannini R, Dell'Omodarme M, Fontanini G, Pacini F, Danesi R, Miccoli P and Toniolo A: Suppression of Fas expression and down-regulation of Fas ligand in highly aggressive human thyroid carcinoma. Lab Invest 80: 1413-1419, 2000.

18. Muraki Y, Tateishi A, Seta C, Fukuda J, Haneji T, Oya R, Ikemura $\mathrm{K}$ and Kobayashi N: Fas antigen expression and outcome of oral squamous cell carcinoma. Int J Oral Maxillofac Surg 29: 360-365, 2000.

19. Hahne M, Rimoldi D, Schroter M, Romero P, Schreier M, French LE, Schneider P, Bornand T, Fontana A, Lienard D, Cerottini J and Tschopp J: Melanoma cell expression of Fas (Apo-1/CD95) ligand: implications for tumor immune escape. Science 274: 1363-1366, 1996. 
20. Krueger A, Baumann S, Krammer PH and Kirchhoff S: FLICEinhibitory proteins: regulators of death receptor-mediated apoptosis. Mol Cell Biol 21: 8247-8254, 2001.

21. Ryu BK, Lee MG, Chi SG, Kim YW and Park JH: Increased expression of cFLIPL in colonic adenocarcinoma. J Pathol 194: 15-19, 2001.

22. Djerbi M, Screpanti V, Catrina AI, Bogen B, Biberfeld P and Grandien A: The inhibitor of death receptor signaling. FLICEinhibitory protein defines a new class of tumor progression factors. J Exp Med 190: 1025-1032, 1999.
23. Medema JP, de Jong J, van Hall T, Melief CJ and Offringa R: Immune escape of tumors in vivo by expression of cellular FLICE-inhibitory protein. J Exp Med 190: 1033-1038, 1999.

24. Kataoka T, Budd RC, Holler N, Thome M, Martinon F, Irmler M, Burns K, Hahne M, Kennedy N, Kovacsovics M and Tschopp J: The caspase- 8 inhibitor FLIP promotes activation of NF-kappaB and Erk-signaling pathways. Curr Biol 10: 640-648, 2000. 